\title{
Microsphere-Based Osteochondral Scaffolds Carrying Opposing Gradients Of Decellularized Cartilage And Demineralized Bone Matrix
}

\author{
Vineet Gupta ${ }^{\dagger}$, Dina V. Lyne ${ }^{\ddagger}$, Amy D. Laflin ${ }^{\ddagger}$, Taylor A. Zabel ${ }^{\S}$, Marilyn Barragan ${ }^{\S}$, Joshua T.

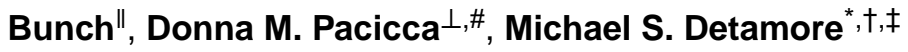 \\ †Bioengineering Graduate Program, University of Kansas, Lawrence, Kansas, United States \\ ¥Department of Chemical and Petroleum Engineering, University of Kansas, Lawrence, Kansas, \\ United States \\ $\S$ Department of Molecular Biosciences, University of Kansas, Lawrence, Kansas, United States \\ "Department of Orthopaedic Surgery, University of Kansas Medical Center, Kansas City, Kansas, \\ United States \\ ${ }^{\perp}$ Division of Orthopaedic Surgery, Children's Mercy Hospital, Kansas City, Missouri, United States \\ \#School of Medicine, University of Missouri-Kansas City, Kansas City, Missouri, United States
}

\section{Abstract}

\begin{abstract}
Extracellular matrix (ECM) "raw materials" such as demineralized bone matrix (DBM) and cartilage matrix have emerged as leading scaffolding materials for osteochondral regeneration owing to their capacity to facilitate progenitor/resident cell recruitment, infiltration, and differentiation without adding growth factors. Scaffolds comprising synthetic polymers are sturdy yet generally lack cues for guiding cell differentiation. We hypothesized that opposing gradients of decellularized cartilage (DCC) and DBM in polymeric microsphere-based scaffolds would provide superior regeneration compared to polymer-only scaffolds in vivo. Poly(D,L-lactic-co-glycolic acid) (PLGA) microsphere-based scaffolds were fabricated, either with opposing gradients of DCC and DBM encapsulated (GRADIENT) or without DCC and DBM (BLANK control), and implanted into rabbit osteochondral defects in medial femoral condyles. After 12 weeks, gross morphological evaluation showed that the repair tissue in about $30 \%$ of the implants was either slightly or significantly depressed, hinting toward rapid polymer degradation in scaffolds from both of the groups. Additionally, no differences were observed in gross morphology of the repair tissue between the BLANK and GRADIENT groups. Mechanical testing revealed no significant differences in model parameter values between the two groups. Histological observations demonstrated that the repair tissue in both of the groups was fibrous in nature with the cells
\end{abstract}

\footnotetext{
*Corresponding Author detamore@ku.edu. Phone: (785) 864-4943.

Supporting Information

The Supporting Information is available free of charge on the ACS Publications website at DOI: 10.1021/acsbiomaterials.6b00071. Figure S1, image of implant placed into induced rabbit medial femoral condyle osteochondral defect; Figure S2, histological and immunohistochemical (IHC) staining images for the BLANK implant along with their histological scores; Figure S3, histological and IHC staining images for the GRADIENT implants along with their histological scores (PDF)

The authors declare no competing financial interest.
} 
demonstrating notable proliferation and matrix deposition activity. No adverse inflammatory response was observed in any of the implants from the two groups. Overall, the results emphasize the need to improve the technology in terms of altering the DBM and DCC concentrations, and tailoring the polymer degradation to these concentrations.

\section{Graphical Abstract}
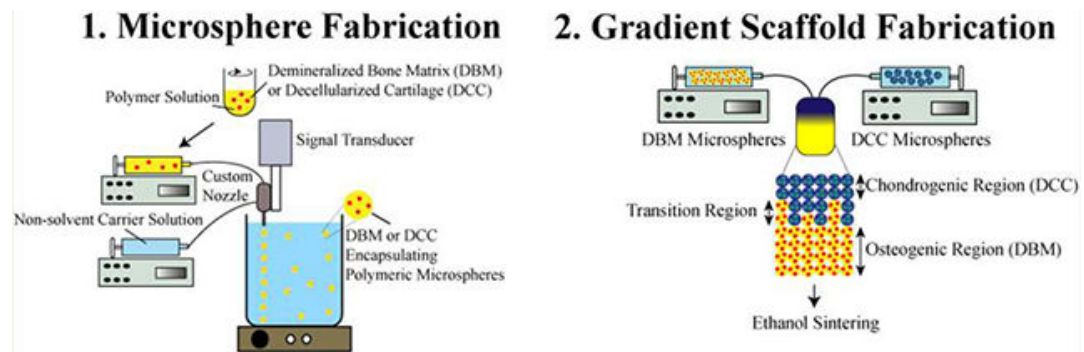

3. Osteochondral Implantation

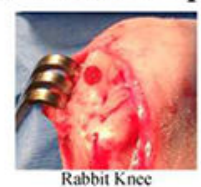

\section{Keywords}

decellularized cartilage; demineralized bone matrix; gradient scaffolds; microsphere-based scaffolds; osteochondral regeneration

\section{INTRODUCTION}

Cartilage injuries are difficult to treat because of the limited capacity of cartilage to heal.

Even though the osteochondral tissue consists of dissimilar tissues, cartilage and bone, it is known that repair of cartilage is associated with the repair of subchondral bone. ${ }^{1}$

Additionally, there are several advantages of growing cartilage and bone close to one another. Several biochemical and biomechanical cues involved in the commitment of progenitor cells to the osteogenic or chondrogenic phenotypes are related to each other. ${ }^{2}$ Therefore, several regenerative medicine strategies for osteochondral repair are focused on regenerating both bone and cartilage simultaneously in a plethora of designs in which stratified and continuously graded designs have emerged as the frontrunners. ${ }^{3}$ Our research group has shown that microsphere-based scaffolds containing opposing gradients of bioactive cues can direct the differentiation of surrounding progenitor cells simultaneously and regionally toward osteogenesis and chondrogenesis. ${ }^{4}$ Moreover, we have demonstrated that these microsphere-based gradient scaffolds can lead to promising osteochondral repair when implanted in vivo in rabbits. ${ }^{5}$

In the past few years, there has been an increased interest in fabricating scaffolds from extracellular matrices (ECM) for regenerative medicine. ${ }^{6}$ For osteochondral regeneration, cartilage matrix and demineralized bone matrix (DBM) have widely gained attention because of their ability to influence resident cell behavior, such as migration, proliferation 
and differentiation. ${ }^{7}$ Both cartilage matrix and DBM allow for constructive remodeling by providing tissue-specific raw materials (i.e., bioactive signals and building blocks). ${ }^{8} \mathrm{DBM}$ is commonly used as a bone graft material and presently there are about 25 different DBM products commercially available in the market. ${ }^{9}$ Cartilage matrix, on the other hand, has started to gain considerable attention in the past few years for cartilage regeneration, ${ }^{10}$ with an emphasis on native tissue-derived, decellularized cartilage (DCC). ${ }^{11}$ Scaffolds derived from DBM and DCC have shown great promise within the field of osteochondral regeneration in part because of their ability to guide cell differentiation and provide raw materials for neo-tissue formation. However, various processing reagents used to obtain DBM or DCC often compromise their mechanical performance. ${ }^{7,8}$

The goal of the current study was to fabricate a scaffold that possesses instructive properties of ECM-based materials to guide cell differentiation and mechanical integrity of strong synthetic materials to support joint function during tissue regeneration. Furthermore, we have previously demonstrated that gradient microsphere-based scaffolds can influence cell behavior and stimulate them to secrete tissue-specific ECM for promoting osteochondral repair. ${ }^{4 a, 5,12}$ Our hypothesis was that microsphere-based scaffolds containing gradients of DBM and DCC would lead to regionalized tissue regeneration following in vivo implantation into rabbit osteochondral defects and outperform the "blank" (no DBM or DCC) microsphere-based scaffolds. To test our hypothesis, we fabricated poly(D,L-lactic acid-co-glycolic acid) (PLGA) microsphere-based scaffolds encapsulating DCC and DBM in opposing gradients, and grafted them into induced femoral condyle osteochondral defects in rabbit knees for 12 weeks. Additionally, PLGA-only microsphere-based scaffolds were also implanted into the contralateral knees of each rabbits as a control.

\section{MATERIALS AND METHODS}

\section{Materials.}

All chemicals for the decellularization process were obtained from Sigma-Aldrich (St.

Louis, MO) unless otherwise specified. PLGA (50:50 lactic acid:glycolic acid ratio, acid end group) with an intrinsic viscosity (i.v.) of $0.37 \mathrm{dL} / \mathrm{g}$, was procured from Evonik Industries (Essen, Germany). Human demineralized bone matrix (DBM) pellets were received from Biomet, Inc. (Warsaw, IN). Two porcine knees obtained from one Berkshire hog (castrated male, age around 7-8 months, and weight $120 \mathrm{~kg}$ ) were bought from Bichelmeyer Meats, Kansas City, KS. Ten New Zealand White rabbits of age 6 months were obtained from Harlan Laboratories (Indianapolis, IN) following the University of Kansas IUCAC procedures (Protocol \#175-21).

\section{Cartilage Harvest and Decellularization.}

Articular cartilage was harvested from hip and knee joints and stored overnight at $-20{ }^{\circ} \mathrm{C}$. The cartilage was later thawed, and coarsely cryo-ground into pellets. Decellularization of the cartilage pellets was performed using our previously described method. ${ }^{10 \mathrm{c}, 13}$ 


\section{Microsphere Fabrication.}

Prior to microsphere fabrication, both the decellularized cartilage (DCC) and DBM pellets were first ground into a fine powder using a freezer-mill (SPEX SamplePrep, Metuchen, $\mathrm{NJ}$ ), freeze-dried, and then sifted through a $45 \mu \mathrm{m}$ mesh (Thermo-Fisher Scientific, Waltham, MA). Three distinct kinds of microspheres were made: (i) PLGA-only microspheres (BLANK), (ii) DBM encapsulated PLGA microspheres (DBM), and (iii) DCC encapsulated PLGA microspheres (DCC). For fabricating DBM and DCC containing microspheres, $2 \% \mathrm{w} / \mathrm{v}$ DBM or DCC was added to $18 \% \mathrm{w} / \mathrm{v}$ PLGA dissolved in methylene chloride, respectively. Using the PLGA, PLGA-DBM, and PLGA-DCC emulsions, microspheres with mean diameters ranging from 240 to $270 \mu \mathrm{m}$ were fabricated using a technology (Figure 1) we have previously described. $4,5,12,13 \mathrm{c}, 14$

\section{Scaffold Fabrication.}

Two scaffold types were evaluated in the study and were labeled in accordance with their microsphere composition as BLANK and GRADIENT. The BLANK scaffolds were fabricated by stacking the "blank" (PLGA-only) microspheres into a cylindrical plastic mold (diameter $\sim 3.6 \mathrm{~mm}$ ) lined with a filter (particle retention $>10 \mu \mathrm{m}$ ) at the bottom, followed by ethanol-acetone (95:5 v/v) sintering for $55 \mathrm{~min} .4,12 \mathrm{a}, \mathrm{c}, 13 \mathrm{c}, 14 \mathrm{~b}, \mathrm{c}$ The gradient ("GRADIENT group) scaffolds were fabricated using the technology from our previous studies. $4,5,12,14 \mathrm{c}, \mathrm{d}$ The dimensions for both the BLANK and GRADIENT scaffolds were $3.5-3.6 \mathrm{~mm} \times 2 \mathrm{~mm}$ (diameter $\times$ height). The BLANK scaffolds were homogenous whereas the profile for the GRADIENT scaffolds was linear, where the top quarter consisted of DCC microspheres $(0.5$ $\mathrm{mm})$, the middle quarter $(0.5 \mathrm{~mm})$ was a progression from DCC to DBM microspheres, and the bottom half $(1 \mathrm{~mm}$ ) comprising DBM microspheres (Figure 2). Preceding animal implantation, the scaffolds from both the groups were sterilized via exposure to ethylene oxide for $12 \mathrm{~h}$.

\section{Surgical Procedure.}

All animal experiments were approved by the IACUC of the University of Kansas (protocol \#175-21). Analgesic and antibiotic were administered preoperatively. Before the procedure, rabbits were also injected with lactated ringer or saline bolus (150-200 cc) subcutaneously at multiple injection sites around the neck and shoulders. Anesthesia was induced using a mixture of ketamine $(40 \mathrm{mg} / \mathrm{kg}$ ) and xylazine $(5 \mathrm{mg} / \mathrm{kg})$, and was maintained via $2-4 \%$ isoflurane inhalation throughout the surgical procedure. ${ }^{12 \mathrm{~b}}$ Aseptic techniques were used for the implantation procedures. A medial parapatellar approach was made, sufficient to allow exposure of the medial condyle. The patella was held laterally as the knee was hyperflexed. A pilot hole was drilled through the cartilage and the subchondral bone in the central loadbearing region of the medial condyle using a $1.5 \mathrm{~mm}$ drill bit. A $3.5 \mathrm{~mm}$ drill bit with depth gauge was used to widen the defect to $3.5 \mathrm{~mm}$ diameter and $2 \mathrm{~mm}$ depth. Afterward, one of the two engineered plugs, either BLANK or GRADIENT, was press fitted into the defect (Figure S1). ${ }^{5 b, 12 b}$ Some plugs needed reasonable force to push them down into the defects because of dissimilarities in diameters of scaffolds and drill bits, culminating in some implant crumbling. Approximately 5\% implant crumbling was noticed in both Rabbit 1 and Rabbit 6 right knees, and 10-15\% implant crumbling in Rabbit 6 left knee. After press- 
fitting the implant, the joint was then irrigated with sterile saline, and the patella carefully reduced into the trochlear groove so as to avoid any disturbance to the implant. The knee arthrotomy was closed with an absorbable suture, and the skin bound with a nonabsorbable suture. Identical procedure was carried out on the contralateral knee but with the alternate plug implanted (Table 1). After both procedures were finished, rabbits were returned to their cages. Analgesics were administered as needed based on pain assessment. The animals were allowed unrestricted movement and weight-bearing postoperatively.

\section{Gross Morphological Assessment.}

After 12 weeks, all of the implants were graded blindly by three different coauthors based on the scoring criteria described previously. ${ }^{12 b, d}$ Thereafter, the joints were photographed and processed for mechanical testing or histology.

\section{Mechanical Testing.}

After retrieval, eight femurs (Table 1) were wrapped in gauze, soaked in a protease inhibitor solution, ${ }^{12 \mathrm{~d}, 15}$ and stored at $-20{ }^{\circ} \mathrm{C}$ until the day of testing. At the day of the testing, the femurs were first thawed and then the medial femoral condyles (MFCs) were carefully separated from the rest of the tissue with a hand-held hacksaw. Each MFC was then glued to a stage, placed in a custom bath and later submerged in the protease inhibitor solution, which was maintained at $37{ }^{\circ} \mathrm{C} .{ }^{12 \mathrm{~d}}$ Cartilage thickness measurement and unconfined indentation stress relaxation tests were performed using Instron 5848 Microtester (Canton, MA) as previously described. ${ }^{12 \mathrm{~d}, 16}$ For stress relaxation testing, central region of the graft site was compressed to $10 \%$ strain (at $0.1 \% / \mathrm{s}$ ) with a spherical stainless steel indenter (diameter $1.5 \mathrm{~mm}$ ), and was later allowed to relax for $1000 \mathrm{s.}^{12 \mathrm{~d}}$

\section{Finite Element Analysis.}

The unconfined compression stress relaxation data was curve-fitted and the indentercartilage contact was modeled using the open-source program FEBio. ${ }^{17}$ The cartilage was modeled as a biphasic material comprising of a porous extracellular matrix described by a solid mixture of a neo-Hookean ground matrix and a continuous, random distribution of fibril bundles. ${ }^{12 \mathrm{~d}, 18}$ The model consisted of five parameters: Elastic modulus $(E)$ and Poisson's ratio $(\nu)$, the fibril modulus $\left(k_{\mathrm{si}}\right)$, the power-law exponent $(\beta)$, and the hydraulic permeability (k), out of which curve-fitting was performed on $E, v, k_{\mathrm{si}}$, and $k$ as " $\beta$ " was assigned a value of $2 .{ }^{12 \mathrm{~d}, 18 \mathrm{a}}$ The indenter-specimen contact was modeled using an impermeable spherical indenter (diameter $1.5 \mathrm{~mm}$ ) and a cylindrical disk of tissue $(6 \mathrm{~mm}$ in diameter and $1 \mathrm{~mm}$ in height) as we have previously described in detail. ${ }^{12 \mathrm{~d}}$ The model tissue diameter was chosen to be 4 times the indenter diameter to simulate the indentation experiment where the cartilage radius is much larger than the indenter radius.

\section{Histological and Immunohistochemical (IHC) Analyses.}

Eight femurs (Table 1) were decalcified in $0.5 \mathrm{~N} \mathrm{HCl}$ with $0.1 \%$ gluteraldehyde for 9 days at $4{ }^{\circ} \mathrm{C}$ with the decalcification solution replaced every $48 \mathrm{~h} .{ }^{19}$ The femurs were then rinsed and defatted in $70 \%$ and $100 \%$ ethanol for $24 \mathrm{~h}$ each. ${ }^{19}$ Thereafter, the femurs were cryoprotected in 5 and $10 \%$ sucrose in PBS for $2 \mathrm{~h}$ each, followed by $20 \%$ sucrose for $16 \mathrm{~h}$ 
at room temperature. ${ }^{19}$ The femurs were then equilibrated in optimal cutting temperature embedding medium (OCT, Tissue-Tek, Torrance, CA) overnight at $37{ }^{\circ} \mathrm{C}$ and then frozen at $-20{ }^{\circ} \mathrm{C}$. The frozen tissue blocks were cut into $10 \mu \mathrm{m}$ thick sections with a cryostat (Micron HM-550 OMP, Vista, CA). The sections were stained using hematoxylin (cell nuclei) and eosin (cytoplasm); safranin-O/fast green (glycosaminoglycans); and Sudan black for residual polymer. Histological scoring was carried out by three independent coauthors using a modified O'Driscoll scoring system. ${ }^{12 \mathrm{~d}, 20}$, The sections from all of the implants were stained for the presence of collagen types I \& II via immunohistochemistry (IHC). IHC for one implant in the GRADIENT group was not performed, as an adequate number of sections could not be obtained. Mouse monoclonal primary antibodies (Thermofisher Scientific, Waltham, MA) against collagen type I (1:200 dilution) and collagen type II (1:250 dilution) were used for IHC. Following incubation with primary antibody, the tissue section was incubated with the biotinylated secondary antibody followed by the $\mathrm{ABC}$ complex (Vector Laboratories, Burlingame, CA). The antibodies were visualized with the diaminobenzidine (DAB) substrate according to the manufacturer's protocol (Vector Laboratories). ${ }^{14 \mathrm{c}}$

\section{Statistical Analyses.}

An unpaired $t$ test performed via GraphPad Prism 6 statistical software (GraphPad Software, Inc., La Jolla, CA) was used to compare the two study groups, where $p<0.05$ was considered significant. All the quantitative results are reported as average \pm standard deviation.

\section{RESULTS}

Tissue Decellularization.

The DNA amount of the native cartilage was lowered by $44 \%(p<0.05)$ following decellularization. The GAG and hydroxyproline contents were both reduced by $23 \%$ ( $p<$ $0.05)$.

\section{Postsurgical Course.}

One rabbit (with the BLANK implant in the right knee and the GRADIENT implant in the left knee) was euthanized prematurely at 2 weeks due to luxation at L6-L7 caused by sudden jumping in the cage. At 7 weeks, another rabbit (with the BLANK implant in the left knee and the GRADIENT implant in the right knee) was euthanized because of chronic lameness. The premature euthanasia of two rabbits brought the sample number down to $n=8$ for gross morphological analysis, $n=4$ for mechanical testing, and $n=4$ for histology and IHC in both the BLANK and GRADIENT groups. All of the other rabbits continued to show normal behavior and movement during the 12 -week period.

\section{Gross Morphological Assessment.}

Visual examination of the joint surface at the time of tissue retrieval revealed no signs of inflammation or infection in any of the rabbits. Additionally, the synovial fluid had a normal color and no signs of degeneration were noted on the opposing joint surfaces. Figure 3 depicts the morphological scores and the amount of repair tissue present at the defect site in both the BLANK and the GRADIENT groups. Figure 4 shows the representative gross 
morphological images of the implants receiving highest, mean, and lowest morphological scores in both the BLANK and the GRADIENT groups.

The average morphological score in the BLANK group was $6.0 \pm 0.5$ out of a maximum possible score of 10 with a highest score of 8.0 while the only BLANK implant that crumbled during implantation received a score of 3.3. All of the rabbits that received the BLANK implants had more than $80 \%$ of the defect area filled with repair tissue, except for two that had 63 and $55 \%$ of the defect area filled with the repair tissue. All of the animals receiving the BLANK implants (except for one) had intermediate smoothness of the repair tissue, with half of them showing complete edge integration with the native tissue. Six of the implants in the BLANK group had slightly depressed repair tissue, with one implant having completely flush and one having significantly depressed surface degree of filling. Seventyfive percent of the animals in the BLANK group had translucent regenerated tissue, whereas the remaining $25 \%$ had an opaque appearance of the repair tissue.

The GRADIENT group average morphological score was $5.2 \pm 0.8$ out of 10 , with the highest score being 8.7 in the group. The GRADIENT implants that crumbled during implantation received average scores of 3.3 and 1.3. The amount of repair tissue present in the GRADIENT group ranged from 7 to $100 \%$ with an average of $61 \pm 11 \%$. No statistically significant differences were observed in either the average morphological scores or the amount of repair tissue between the BLANK and the GRADIENT groups. Almost all of the animals in the GRADIENT group showed complete integration of the repair tissue with the native tissue, with two animals showing partial integration and one showing no integration at all. $63 \%$ of the rabbits receiving the GRADIENT implant had high or intermediate smoothness of the repair tissue, whereas the remaining $37 \%$ had a rough appearance of the regenerated tissue. Half of the animals in the GRADIENT group had either completely flush or slightly depressed regenerated tissue, whereas the other half had significantly depressed repair tissue. All the animals in the GRADIENT group had either translucent or opaque appearance of the repair tissue.

\section{Mechanical Testing and Finite Element Analysis.}

The elastic modulus $(E)$, fiber modulus $\left(k_{\mathrm{si}}\right)$, permeability $(k)$, and Poisson's ratio $(v)$ are depicted in Figure 5. The average elastic moduli, obtained from curve fitting the indentation stress relaxation data, for the BLANK and GRADIENT groups were $0.53 \pm 0.61 \mathrm{MPa}$ and $1.1 \pm 1.8 \mathrm{MPa}$, respectively. The fiber moduli for the BLANK and the GRADIENT groups were found to be $1.1 \pm 1.9 \mathrm{MPa}$ and $0.53 \pm 0.84 \mathrm{MPa}$, respectively. No statistically significant differences were observed in the elastic and fiber moduli between the BLANK and GRADIENT groups. The BLANK group had an average hydraulic permeability of 1.72 $\pm 1.09 \times 10^{-15} \mathrm{~m}^{4} / \mathrm{N}$.s and the GRADIENT group had a permeability of $1.74 \pm 1.57 \times 10^{-15}$ $\mathrm{m}^{4} /(\mathrm{N} \mathrm{s})$. The Poisson's ratios for the BLANK and GRADIENT groups were $0.17 \pm 0.15$ and $0.043 \pm 0.051$, respectively. No statistically significant differences were observed in the hydraulic permeabilities and Poisson's ratios between the BLANK and GRADIENT groups. 


\section{Histology and Immunohistochemistry (IHC).}

Both the BLANK and GRADIENT groups had an average histological score close to 10 (out of a maximum possible 28) with the BLANK group having a mean score of $7.6 \pm 0.9$ and the GRADIENT group having a mean score of $7.4 \pm 0.6$ (difference not statistically significant).

The representative histological and IHC images from the implants in the BLANK and GRADIENT groups are depicted in Figure 6, whereas images from all the implants in both the groups can be found within the Figure S2 and S3. The BLANK implant showed significantly depressed repair tissue at the defect site. The regenerated tissue largely resembled fibrous tissue with most of the cells having a flat appearance. A few cells at the surface of the defect site appeared to have chondroblastlike morphology. The regenerated tissue showed severe disruptions; however, no cysts were noted in the repair tissue. The defect site did not stain for Safranin-O while the surrounding native tissue showed slight Safranin-O staining. Toward the subchondral bone region, the defect area showed signs of osteoblastic activity with minimal bone regeneration and presence of some blood vessels. No adverse inflammatory response was observed in the defect area. The repair tissue stained positively for collagen I, whereas collagen II staining was predominantly absent from the repair tissue. The GRADIENT implant also possessed repair tissue that was significantly depressed compared to the native tissue. The repair tissue in the cartilage part of the defect area resembled fibrocartilage with the cells demonstrating good proliferative activity. The regenerated cartilage was partially bonded with the native tissue on one side of the defect. The adjacent native cartilage showed some signs of degenerative changes as indicated by moderate hypocellularity in the surrounding native cartilage. The defect area showed no to minimal signs of subchondral bone reconstruction, although some blood vessels were noticed in the region. A mild inflammatory response was also observed as indicated by the presence of a few giant multinucleated cells. The tissue in the defect area stained positively for collagen I. Slight collagen II staining was also observed in the areas adjacent to the defect edges.

Figure 7 shows the Sudan Black staining images for the implants that received the highest and lowest histological scores in both the BLANK and GRADIENT groups. Only a few areas of intense staining (depicted by arrow heads) were observed in all of the implants with most of the defect site showing no to mild staining.

\section{DISCUSSION}

The present study for the first time evaluated the in vivo response of microencapsulating demineralized bone matrix (DBM) and cartilage matrix in polymeric microsphere-based scaffolds toward osteochondral repair. Moreover, we are the first group to incorporate DBM in microsphere-based scaffolds for the bone region of the scaffold, whereas the other groups have banked on calcium phosphates for engineering the bone tissue. ${ }^{21}$

From the gross morphological evaluation, it was observed that in 50\% of the total implants (both of the groups combined) about one-fourth of the defect area was empty. Additionally, 5 out of the total 16 implants had either slightly or significantly depressed repair tissue. No 
statistically significant differences were observed in the BLANK and GRADIENT groups in the amount of defect area fill; however, the average fill area in the GRADIENT group was lower than the average fill area in the BLANK group. Moreover, 50\% of the implants in the GRADIENT group had significantly depressed regenerated tissue whereas the corresponding number in the BLANK group was $13 \%$. The presence of empty area and the observance of depressed tissue might have resulted from the rapid degradation of polymer, with even faster degradation occurring in the GRADIENT group. Our prior work involving gradient microsphere-based scaffolds for osteochondral regeneration in rabbits with a comparable polymer (i.v. $0.34-0.36 \mathrm{dL} / \mathrm{g}$ ) as we have used in the current study, showed a defect fill of more than $95 \%$ in all of the implants at 12 weeks. ${ }^{12 b}$ The amount of repair tissue present in defect site in the current study was about 80 and $60 \%$ for the BLANK and GRADIENT groups, respectively. Lower amounts of repair tissue in the current study most likely resulted from the rapid scaffold degradation, which is a direct consequence of polymer degradation. It is believed that DBM and DCC encapsulation might have affected microsphere morphology, which might have resulted in faster degradation of the polymer; however, no analysis was done to assess polymer degradation, which can be a subject matter for subsequent studies. We have formerly observed that encapsulation of raw materials such as chondroitin sulfate and DCC result in minute pores on the surface of PLGA microspheres that can accelerate polymer degradation. ${ }^{12 a, c, 13 c, 22}$

Increasing concentrations of raw materials on the one hand might accelerate but on the other might provide momentum to tissue regeneration for impeding the deleterious effects caused by rapid polymer degradation. Furthermore, it was observed that some of the implants crumbled during implantation that might have further accelerated scaffold degradation. The implants that crumbled in both of the groups received the lowest morphological scores; further suggesting that implant crumbling might have also affected scaffold degradation. The implant crumbling might have resulted due to slight mismatch between scaffold and defect diameters. The scaffold diameter was intentionally kept slightly larger than the defect diameter to allow for press-fitting of the implant. ${ }^{12 \mathrm{~b}}$ In addition, variations in force applied due to the manual press-fitting approach might have caused some implants to crumble, which can be avoided by using an automated approach (maybe a delivery device) for implanting the scaffold. The average morphological scores in the BLANK and GRADIENT groups were similar to each other, although there were some differences observed in the macroscopic properties of the repair tissue between the two groups. The repair tissue in the GRADIENT group had better edge integration with the peripheral native tissue than the edge integration observed in the BLANK group. On the other hand, the repair tissue smoothness in the BLANK group surpassed the smoothness of the repair tissue in the GRADIENT group.

The elastic moduli, fiber moduli, permeability, and Poisson's ratio values of the repair tissue in the BLANK and GRADIENT groups fell within the previously reported values of these parameters. ${ }^{15,23}$ Julkunen et al. ${ }^{23}$ reported the elastic moduli, fiber moduli, and permeability values of healthy rabbit articular cartilage samples harvested from 0 to 18 month old female animals to be $0.66 \pm 0.50 \mathrm{MPa}, 19.6 \pm 20.2 \mathrm{MPa}$, and $0.579 \pm 0.319 \times 10^{-15} \mathrm{~m}^{4} /(\mathrm{N} \mathrm{s})$, respectively. Athanasiou et al. ${ }^{15}$ reported the Poisson's ratios of $0.197 \pm 0.094$ for rabbit articular cartilage. It was reported that the Poisson's ratio of cartilage can be as low as 0.02 
in unconfined compression, but never more than $0.5 .^{18 b, 24}$ In the current study, the Poisson's ratio values appeared to be lower in the GRADIENT group than those observed in the BLANK group, indicating higher apparent compressibility of the tissue and a propensity for more fluid transport in the GRADIENT group. ${ }^{15}$ However, no statistically significant differences were observed in the Poisson's ratios between the BLANK and GRADIENT groups to support that claim.

The histological findings revealed no significant differences in the average histological scores between the BLANK and GRADIENT groups. The cellular morphology observed in the repair tissues in both of the groups resembled those of fibroblasts with the cells demonstrating significant proliferative activity. The defect site in both of the groups was largely filled with a vascularized loose connective tissue; however, some implants showed a presence of a fibrocartilage layer on the top. Additionally, both of the groups showed a mild inflammatory response in the subchondral bone region. The inflammatory response may have been a manifestation of tissue remodeling response that can have profound implications in clinical success of microsphere-based scaffolds. ${ }^{25}$ The presence of premature bone trabeculae in some of the implants in both the groups also hint toward the regeneration or remodeling response in the defect site. However, both the BLANK and GRADIENT groups showed void areas in the subchondral bone region, which indicate toward rapid polymer degradation that may have further led to depressed repair tissue overall in both the groups. Sudan black staining images revealed that the defect sites in both the groups were predominantly absent of residual polymer except for a few small dark-staining spots that indicated minimal leftover polymer or polymer degradation products. The absence of residual polymer in both the BLANK and GRADIENT groups further hint toward rapid polymer degradation in microsphere-based scaffolds that most likely adversely affected the regeneration of the tissue in the defect site.

Results from the current study underscore the significance of determining polymer and raw material concentrations that would further lead to comparable scaffold degradation and in vivo tissue regeneration rates. Even though the polymer selection in the current study was based on our prior in vitro ${ }^{12 \mathrm{c}}$ and in vivo ${ }^{12 \mathrm{~b}}$ iterations with microsphere-based scaffolds, the rapid polymer degradation led to below average performance of these scaffolds in the current study. The rapid polymer degradation, more so in the GRADIENT group, could be ascribed to the encapsulated materials that can alter polymer degradation kinetics in vitro as we have observed previously. ${ }^{22 \mathrm{~b}}$ Monitoring changes to scaffold integrity, structure, and mass in vitro can provide useful information regarding scaffold degradation; however, degradation in vivo is more complicated than in vitro, and in vitro assays are seldom acceptable measures of in vivo scaffold behavior. ${ }^{26}$ Thus, it is to be emphasized that an iterative approach involving in vitro testing and empirical in vivo evaluation, as we have done here, is needed for assessing degradation of these microsphere-based scaffolds.

\section{CONCLUSIONS}

The current study demonstrated that structural integrity of microsphere-based scaffolds is an important parameter than can have significant impact on tissue regeneration. The polymer in both the BLANK and GRADIENT groups degraded quickly and induced a pro- 
inflammatory response, which further resulted in more of a fibrocartilage like repair tissue. The degradation of the polymer in the scaffolds will be vital to tissue regeneration in vivo, where extended degradation could become an obstacle to tissue regeneration and in contrast rapid degradation could have a damaging effect on the regenerating tissue, supposedly the cause of below par regeneration observed in the current study. Thus, we acknowledge that there should be a more detailed look at polymer degradation as a function of encapsulated raw materials for subsequent in vitro and in vivo studies to identify formulations that more closely approximate the tissue regeneration rate in animals and humans. Additionally, emphasis is laid on the need to better match scaffold dimensions with defect dimensions or perhaps employ a scaffold delivery device that can further minimize surgeon-to-surgeon variations during scaffold implantation. Nevertheless, the current study hints that employing gradients of DBM and DCC might be beneficial for osteochondral regeneration as indicated by better integration of the regenerated tissue with the peripheral native tissue, thus providing a motivation for further refining the technology by altering the concentrations of DBM and DCC, and attuning polymer degradation to these concentrations.

\title{
Supplementary Material
}

Refer to Web version on PubMed Central for supplementary material.

\section{ACKNOWLEDGMENTS}

\begin{abstract}
We gratefully acknowledge funding from the National Institute of Arthritis and Musculoskeletal and Skin Diseases of the National Institutes of Health (R01 AR056347). Any views, findings, and suggestions communicated in this article are those of the authors and do not necessarily represent the views of the National Institutes of Health. We also acknowledge funding from the Kansas Bioscience Authority Rising Star Award. In addition, we thank Biomet, Inc (Warsaw, IN) for the generous gift of human demineralized bone matrix pellets. We acknowledge Dr. Gerard Ateshian for his assistance with the finite element analysis. Furthermore, we are grateful to Dr. Neethu Mohan for her contributions to the morphological and histological scoring. Lastly, we thank Dr. Richard Galbraith for his valuable opinion on the histological images of the study implants.
\end{abstract}

\section{REFERENCES}

(1). Lu S; Lam J; Trachtenberg JE; Lee EJ; Seyednejad H; van den Beucken JJJP; Tabata Y; Kasper FK; Scott DW; Wong ME; Jansen JA; Mikos AG Technical Report: Correlation Between the Repair of Cartilage and Subchondral Bone in an Osteochondral Defect Using Bilayered, Biodegradable Hydrogel Composites. Tissue Eng., Part C 2015, 21 (12), 1216-1225.

(2). (a)de Vries-van Melle ML; Narcisi R; Kops N; Koevoet WJ; Bos PK; Murphy JM; Verhaar JA; van der Kraan PM; van Osch GJ Chondrogenesis of mesenchymal stem cells in an osteochondral environment is mediated by the subchondral bone. Tissue Eng., Part A 2013, 20 (1-2), 23-33. [PubMed: 23980750] (b)Gordeladze JO; Djouad F; Brondello J-M; Noël D; Duroux-Richard I; Apparailly F; Jorgensen C Concerted stimuli regulating osteo-chondral differentiation from stem cells: phenotype acquisition regulated by microRNAs. Acta Pharmacol. Sin 2009, 30 (10), 13691384. [PubMed: 19801995] (c)RADIN EL; EHRLICH MG; Chernack R; Abernethy P; PAUL IL; ROSE RM Effect of repetitive impulsive loading on the knee joints of rabbits. Clin. Orthop. Relat. Res 1978, 131, 288-293.

(3). (a)Dormer NH; Berkland CJ; Detamore MS Emerging Techniques in Stratified Designs and Continuous Gradients for Tissue Engineering of Interfaces. Ann. Biomed. Eng 2010, 38 (6), 2121-2141. [PubMed: 20411333] (b)Lopa S; Madry H Bioinspired Scaffolds for Osteochondral Regeneration. Tissue Eng., Part A 2014, 20 (15-16), 2052-2076. [PubMed: 24476065] (c)Shimomura K; Moriguchi Y; Murawski CD; Yoshikawa H; Nakamura N Osteochondral Tissue Engineering with Biphasic Scaffold: Current Strategies and Techniques. Tissue Eng., Part B 2014, 20, 468-476. 
(4). (a)Dormer NH; Singh M; Wang L; Berkland CJ; Detamore MS Osteochondral interface tissue engineering using macroscopic gradients of bioactive signals. Ann. Biomed. Eng 2010, 38 (6), 2167-2182. [PubMed: 20379780] (b)Singh M; Morris CP; Ellis RJ; Detamore MS; Berkland C Microsphere-based seamless scaffolds containing macroscopic gradients of encapsulated factors for tissue engineering. Tissue Eng., Part C 2008, 14 (4), 299-309.

(5). (a)Dormer NH; Busaidy K; Berkland CJ; Detamore MS Osteochondral interface regeneration of rabbit mandibular condyle with bioactive signal gradients. Journal of Oral and Maxillofacial Surgery 2011, 69 (6), e50-e57. [PubMed: 21470747] (b)Dormer NH; Singh M; Zhao L; Mohan N; Berkland CJ; Detamore MS Osteochondral interface regeneration of the rabbit knee with macroscopic gradients of bioactive signals. J. Biomed. Mater. Res., Part A 2012, 100 (1), 162170.

(6). (a)Boccafoschi F; Botta M; Fusaro L; Copes F; Ramella M; Cannas M Decellularized biological matrices: an interesting approach for cardiovascular tissue repair and regeneration. J. Tissue Eng. Regener. Med 2015, DOI: 10.1002/term.2103.(b)Hoshiba T; Lu H; Kawazoe N; Chen G Decellularized matrices for tissue engineering. Expert Opin. Biol. Ther 2010, 10 (12), 17171728. [PubMed: 21058932]

(7). Cheng CW; Solorio LD; Alsberg E Decellularized tissue and cell-derived extracellular matrices as scaffolds for orthopaedic tissue engineering. Biotechnol. Adv 2014, 32 (2), 462-484. [PubMed: 24417915]

(8). Benders KEM; van Weeren PR; Badylak SF; Saris DBF; Dhert WJA; Malda J Extracellular matrix scaffolds for cartilage and bone regeneration. Trends Biotechnol 2013, 31 (3), 169-176. [PubMed: 23298610]

(9). Drosos GI; Kazakos KI; Kouzoumpasis P; Verettas D-A Safety and efficacy of commercially available demineralised bone matrix preparations: a critical review of clinical studies. Injury 2007, 38, S13-S21. [PubMed: 18224733]

(10). (a)Almeida HV; Liu Y; Cunniffe GM; Mulhall KJ; Matsiko A; Buckley CT; O’Brien FJ; Kelly DJ Controlled release of transforming growth factor- $\beta 3$ from cartilage-extra-cellular-matrix-derived scaffolds to promote chondrogenesis of human-joint-tissue-derived stem cells. Acta Biomater. 2014, 10 (10), 4400-4409. [PubMed: 24907658] (b)Badylak SF; Freytes DO; Gilbert TW Extracellular matrix as a biological scaffold material: structure and function. Acta Biomater. 2009, 5 (1), 1-13. [PubMed: 18938117] (c)Beck EC; Barragan M; Tadros MH; Kiyotake EA; Acosta FM; Kieweg SL; Detamore MS Chondroinductive Hydrogel Pastes Composed of Naturally Derived Devitalized Cartilage. Ann. Biomed. Eng 2016, 44, 1863-1880. [PubMed: 26744243] (d)Garrigues NW; Little D; Sanchez-Adams J; Ruch DS; Guilak F Electrospun cartilage-derived matrix scaffolds for cartilage tissue engineering. J. Biomed. Mater. Res., Part A 2014, 102 (11), 3998-4008.(e)Moradi A; Ataollahi F; Sayar K; Pramanik S; Chong P-P; Khalil AA; Kamarul T; Pingguan-Murphy B Chondrogenic potential of physically treated bovine cartilage matrix derived porous scaffolds on human dermal fibroblast cells. J. Biomed. Mater. Res., Part A 2016, 104 (1), 245-256.(f)Schwarz S; Koerber L; Elsaesser AF; Goldberg-Bockhorn E; Seitz AM; Durselen L; Ignatius A; Walther P; Breiter R; Rotter N Decellularized cartilage matrix as a novel biomatrix for cartilage tissue-engineering applications. Tissue Eng., Part A 2012, 18 (21-22), 2195-2209. [PubMed: 22690787]

(11). Sutherland AJ; Converse GL; Hopkins RA; Detamore MS The Bioactivity of Cartilage Extracellular Matrix in Articular Cartilage Regeneration. Adv. Healthcare Mater 2015, 4 (1), 29 39.

(12). (a)Gupta V; Mohan N; Berkland CJ; Detamore MS Microsphere-Based Scaffolds Carrying Opposing Gradients of Chondroitin Sulfate and Tricalcium Phosphate. Front. Bioeng. Biotechnol 2015, 3, 1-15. [PubMed: 25654078] (b)Mohan N; Dormer NH; Caldwell KL; Key VH; Berkland CJ; Detamore MS Continuous gradients of material composition and growth factors for effective regeneration of the osteochondral interface. Tissue Eng., Part A 2011, 17 (21-22), 2845-2855. [PubMed: 21815822] (c)Mohan N; Gupta V; Sridharan B; Sutherland A; Detamore MS The potential of encapsulating "raw materials" in 3D osteochondral gradient scaffolds. Biotechnol. Bioeng 2014, 111 (4), 829-841. [PubMed: 24293388] (d)Mohan N; Gupta V; Sridharan BP; Mellott AJ; Easley JT; Palmer RH; Galbraith RA; Key VH; Berkland CJ; Detamore MS Microsphere-based gradient implants for osteochondral regeneration: a long-term study in sheep. Regener. Med 2015, 10, 709. 
(13). (a)Converse GL; Armstrong M; Quinn RW; Buse EE; Cromwell ML; Moriarty SJ; Lofland GK; Hilbert SL; Hopkins RA Effects of cryopreservation, decellularization and novel extracellular matrix conditioning on the quasi-static and time-dependent properties of the pulmonary valve leaflet. Acta Biomater. 2012, 8 (7), 2722-2729. [PubMed: 22484150] (b)Sutherland AJ; Beck EC; Dennis SC; Converse GL; Hopkins RA; Berkland CJ; Detamore MS Decellularized Cartilage May Be a Chondroinductive Material for Osteochondral Tissue Engineering. PLoS One 2015, 10 (5), e0121966-13. [PubMed: 25965981] (c)Sutherland AJ; Detamore MS Bioactive Microsphere-Based Scaffolds Containing Decellularized Cartilage. Macromol. Biosci 2015, 15 (7), 979-989. [PubMed: 25821206]

(14). (a)Dormer NH; Gupta V; Scurto AM; Berkland CJ; Detamore MS Effect of different sintering methods on bioactivity and release of proteins from PLGA microspheres. Mater. Sci. Eng., C 2013, 33 (7), 4343-4351.(b)Dormer NH; Qiu Y; Lydick AM; Allen ND; Mohan N; Berkland CJ; Detamore MS Osteogenic differentiation of human bone marrow stromal cells in hydroxyapatiteloaded microsphere-based scaffolds. Tissue Eng., Part A 2012, 18 (7-8), 757-767. [PubMed: 21992088] (c)Gupta V; Lyne DV; Barragan M; Berkland CJ; Detamore MS Microsphere-based scaffolds encapsulating tricalcium phosphate and hydroxyapatite for bone regeneration. J. Mater. Sci.: Mater. Med 2016, 27 (7), 1-17. [PubMed: 26610924] (d)Singh M; Dormer N; Salash JR; Christian JM; Moore DS; Berkland C; Detamore MS Three-dimensional macroscopic scaffolds with a gradient in stiffness for functional regeneration of interfacial tissues. J. Biomed. Mater. Res., Part A 2010, 94 (3), 870-876.

(15). Athanasiou KA; Rosenwasser MP; Buckwalter JA; Malinin TI; Mow VC Interspecies comparisons of in situ intrinsic mechanical properties of distal femoral cartilage. J. Orthop. Res 1991, 9 (3), 330-340. [PubMed: 2010837]

(16). Roemhildt ML; Coughlin KM; Peura GD; Fleming BC; Beynnon BD Material properties of articular cartilage in the rabbit tibial plateau. J. Biomech 2006, 39 (12), 2331-2337. [PubMed: 16168420]

(17). Maas SA; Ellis BJ; Ateshian GA; Weiss JA FEBio: finite elements for biomechanics. J. Biomech. Eng 2012, 134 (1), 011005. [PubMed: 22482660]

(18). (a)Ateshian GA; Mauck RL Sliding contact loading enhances the tensile properties of mesenchymal stem cell-seeded hydrogels. Eur. Cell Mater 2012, 24, 29-45. [PubMed: 22791371] (b)Ateshian GA; Rajan V; Chahine NO; Canal CE; Hung CT Modeling the Matrix of Articular Cartilage Using a Continuous Fiber Angular Distribution Predicts Many Observed Phenomena. J. Biomech. Eng 2009, 131 (6), 061003. [PubMed: 19449957] (c)Bonet J Nonlinear Continuum Mechanics for Finite Element Analysis; Cambridge University Press: Cambridge, U.K., 1997. (d)Mow VC; Gibbs MC; Lai WM; Zhu WB; Athanasiou KA Biphasic indentation of articular cartilage-II. A numerical algorithm and an experimental study. J. Biomech 1989, 22 (8-9), 853861. [PubMed: 2613721]

(19). Chevrier A; Rossomacha E; Buschmann MD; Hoemann CD Optimization of histoprocessing methods to detect glycosamino- glycan, collagen type II, and collagen type I in decalcified rabbit osteochondral sections. J. Histotechnol 2005, 28 (3), 165-175.

(20). (a)Frisbie D; Bowman S; Colhoun H; DiCarlo E; Kawcak C; McIlwraith C Evaluation of autologous chondrocyte transplantation via a collagen membrane in equine articular defectsresults at 12 and 18 months. Osteoarthritis and Cartilage 2008, 16 (6), 667-679. [PubMed: 18042409] (b)O'Driscoll SW; Keeley FW; Salter RB Durability of regenerated articular cartilage produced by free autogenous periosteal grafts in major full-thickness defects in joint surfaces under the influence of continuous passive motion. A follow-up report at one year. J. Bone Jt. Surg 1988, 70 (4), 595-606.

(21). (a)Cushnie EK; Khan YM; Laurencin CT Amorphous hydroxyapatite-sintered polymeric scaffolds for bone tissue regeneration: physical characterization studies. J. Biomed. Mater. Res., Part A 2008, 84 (1), 54-62.(b)Lv Q; Nair L; Laurencin CT Fabrication, characterization, and in vitro evaluation of poly(lactic acid glycolic acid)/nano-hydroxyapatite composite microspherebased scaffolds for bone tissue engineering in rotating bioreactors. J. Biomed. Mater. Res., Part A 2009, 91 (3), 679-691.(c)Tahriri M; Moztarzadeh F Preparation, Characterization, and In Vitro Biological Evaluation of PLGA/Nano-Fluorohydroxyapatite (FHA) Microsphere-Sintered Scaffolds for Biomedical Applications. Appl Biochem. Biotechnol 2014, 172 (5), 2465-2479. [PubMed: 24395697] (d)Xu W; Wang L; Ling Y; Wei K; Zhong S Enhancement of compressive 
strength and cytocompatibility using apatite coated hexagonal mesoporous silica/poly(lactic acidglycolic acid) microsphere scaffolds for bone tissue engineering. RSC Adv. 2014, 4 (26), 13495.

(22). (a)Alexis F Factors affecting the degradation and drug-release mechanism of poly(lactic acid) and poly[(lactic acid)-co-(glycolic acid)]. Polym. Int 2005, 54 (1), 36-46.(b)Sridharan B; Mohan N; Berkland CJ; Detamore MS Material characterization of microsphere-based scaffolds with encapsulated raw materials. Mater. Sci. Eng., C 2016, 63, 422-428.

(23). (a)Julkunen P; Harjula T; Iivarinen J; Marjanen J; Seppänen K; Närhi T; Arokoski J; Lammi MJ; Brama PA; Jurvelin JS; Helminen HJ Biomechanical, biochemical and structural correlations in immature and mature rabbit articular cartilage. Osteoarthritis and cartilage/OARS, Osteoarthritis Research Society 2009, 17 (12), 1628-1638.(b)Julkunen P; Harjula T; Marjanen J; Helminen HJ; Jurvelin JS Comparison of singlephase isotropic elastic and fibril-reinforced poroelastic models for indentation of rabbit articular cartilage. J. Biomech 2009, 42 (5), 652-656. [PubMed: 19193381]

(24). Wang CC; Deng J-M; Ateshian GA; Hung CT An automated approach for direct measurement of two-dimensional strain distributions within articular cartilage under unconfined compression. J. Biomech. Eng 2002, 124 (5), 557-567. [PubMed: 12405599]

(25). Londono R; Badylak SF Biologic Scaffolds for Regenerative Medicine: Mechanisms of In vivo Remodeling. Ann. Biomed. Eng 2015, 43 (3), 577-592. [PubMed: 25213186]

(26). Artzi N; Oliva N; Puron C; Shitreet S; Artzi S; Bon Ramos A; Groothuis A; Sahagian G; Edelman ER In vivo and in vitro tracking of erosion in biodegradable materials using noninvasive fluorescence imaging. Nat. Mater 2011, 10 (9), 704. [PubMed: 21857678] 


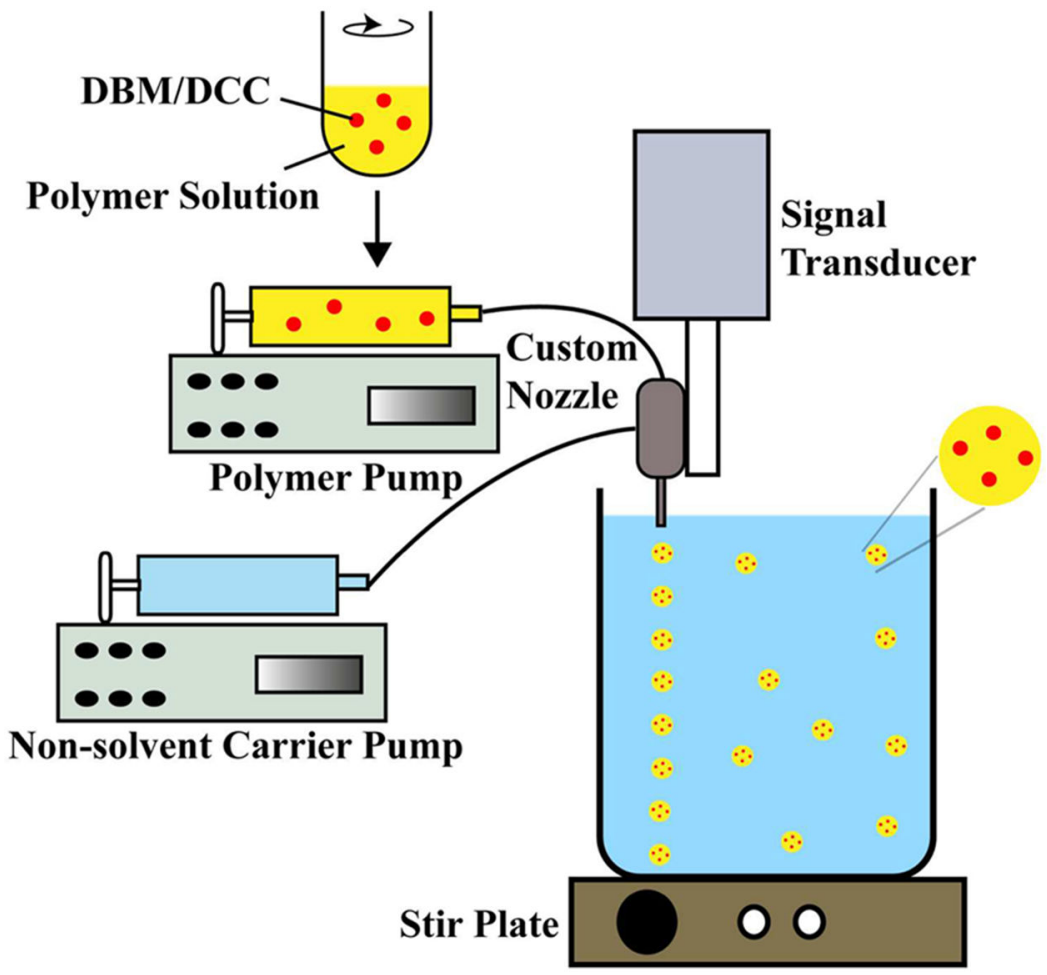

Figure 1.

A schematic representation of microsphere fabrication process. Demineralized bone matrix (DBM) or decellularized cartilage (DCC) encapsulating poly(D,L-lactic-co-glycolic acid) (PLGA) microspheres were fabricating with a DBM/DCC loading of $10 \mathrm{wt} \%$. 
GRADIENT

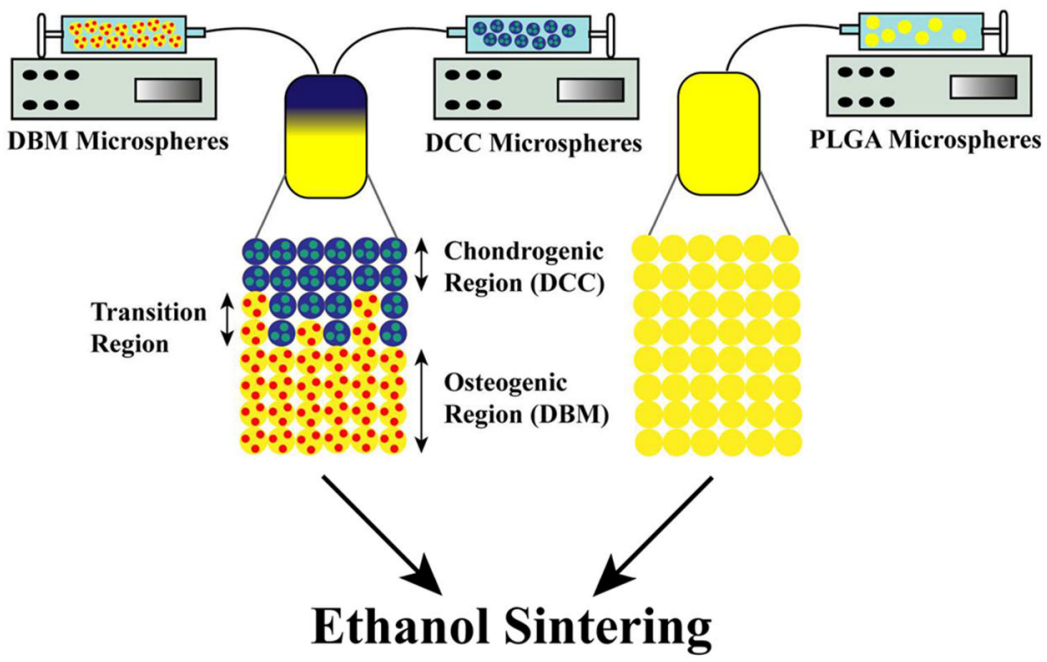

Figure 2.

Schematic of scaffold fabrication process. Demineralized bone matrix (DBM) or decellularized cartilage (DCC) encapsulating poly(D,L-lactic-co-glycolic acid) (PLGA) microspheres were assembled to form continuously graded scaffolds containing opposing gradients of DCC and DBM. 

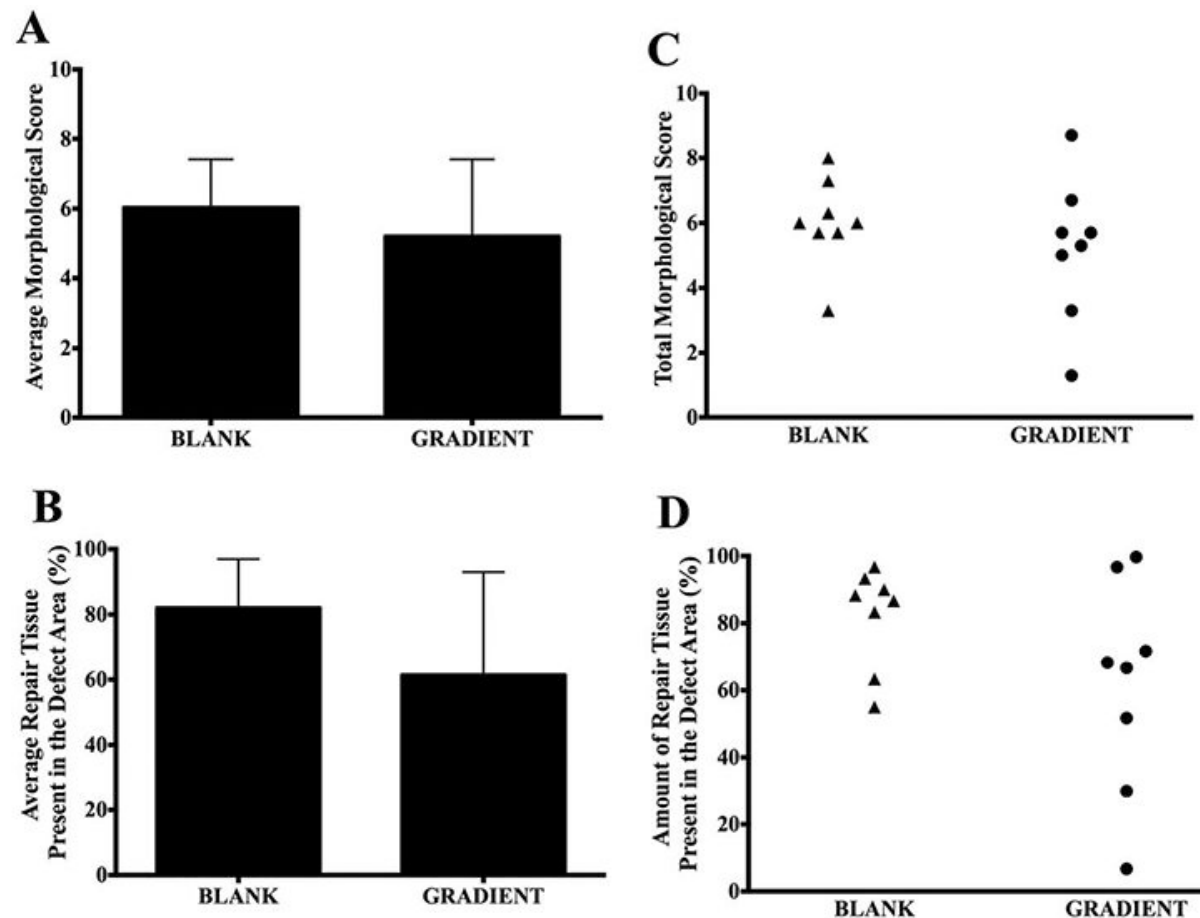

D

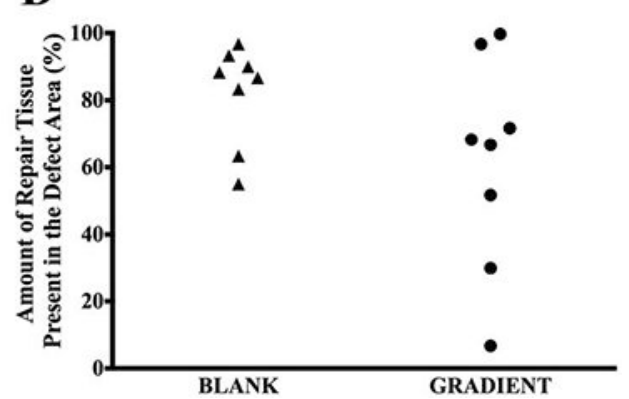

Figure 3.

Gross morphological scores of retrieved joints 12 weeks postimplantation. (A) Average morphological score in the BLANK and GRADIENT groups, (B) average percentage of repair tissue present at the defect site in the BLANK and GRADIENT groups, (C) scatter plot showing total morphological score distribution in the BLANK and GRADIENT groups, and (D) scatter plot showing amount of repair tissue present in the defect site in the BLANK and GRADIENT groups. All values are expressed as the average + standard deviation $(n=$ 8 ). The maximum possible score was 10 . No significant differences in gross morphological scores and amount of repair tissue present were observed between the two groups. The average morphological score in the BLANK group was $6.0 \pm 0.5$ with a highest score of 8.0, whereas the GRADIENT group average morphological score was $5.2 \pm 0.8$ with a highest score of 8.7 in the group. 


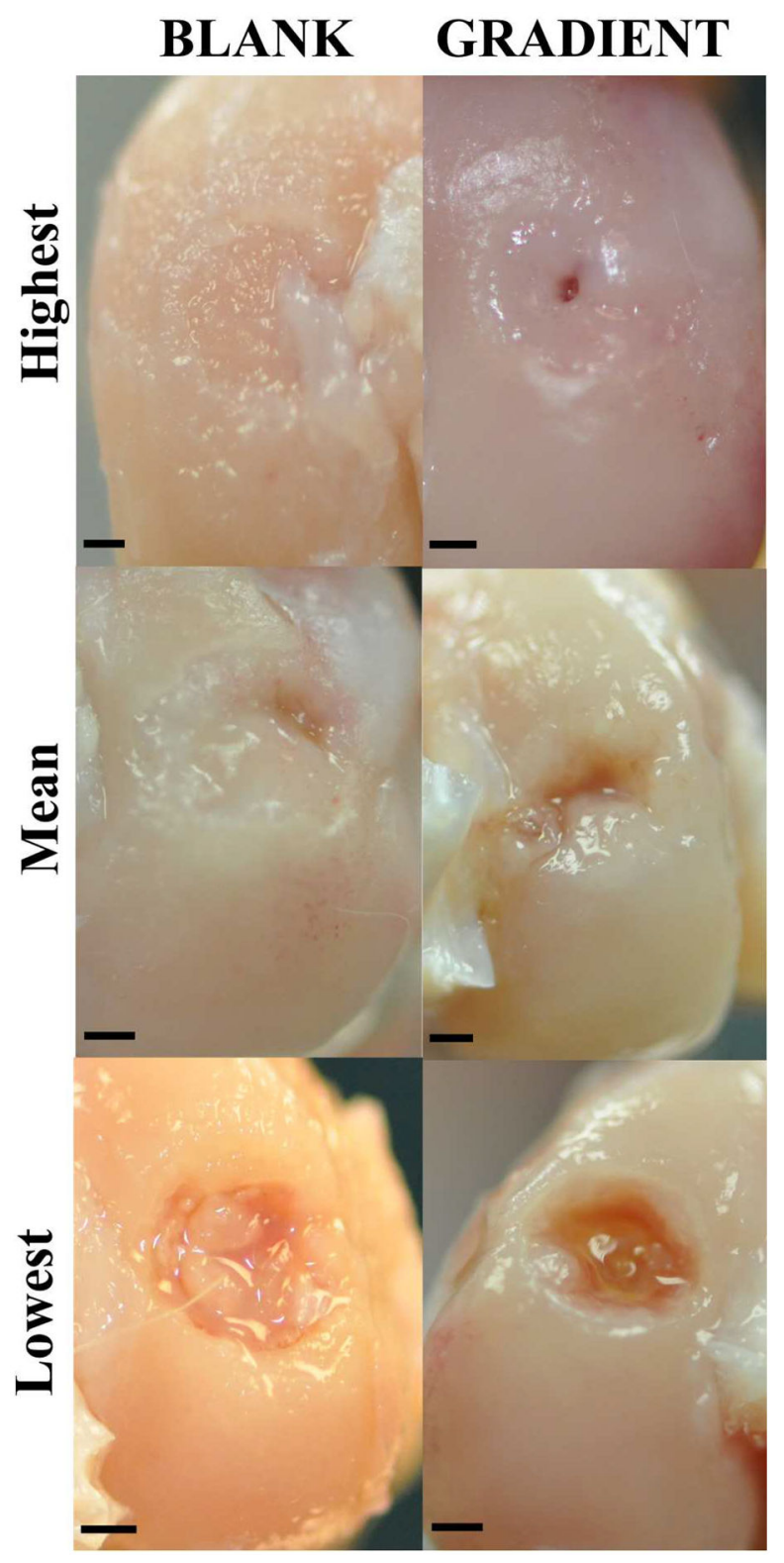

Figure 4.

Representative images for gross morphology of the BLANK and GRADIENT groups at 12 weeks postimplantation. The top row represents images from the implants that received the highest morphological score (BLANK, 8; GRADIENT, 8.7). The middle row represents images from the implants that received the mean morphological score (BLANK, 6; GRADIENT, 5.2). The bottom row represents images from the implants that received the lowest morphological score (BLANK, 3.3; GRADIENT, 1.3). The BLANK group performed better in percent defect fill, whereas the GRADIENT group performed better in edge integration with the native tissue. Scale bars: $1 \mathrm{~mm}$. 
A

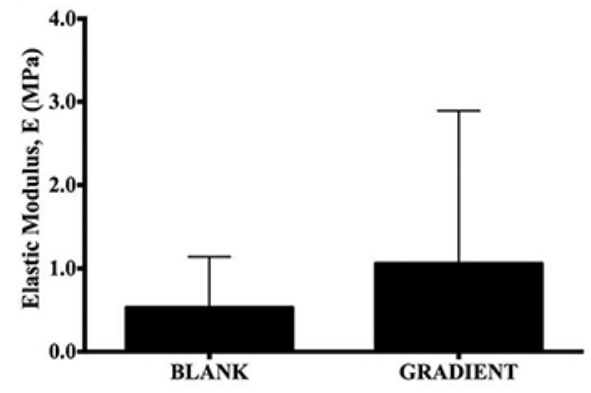

B

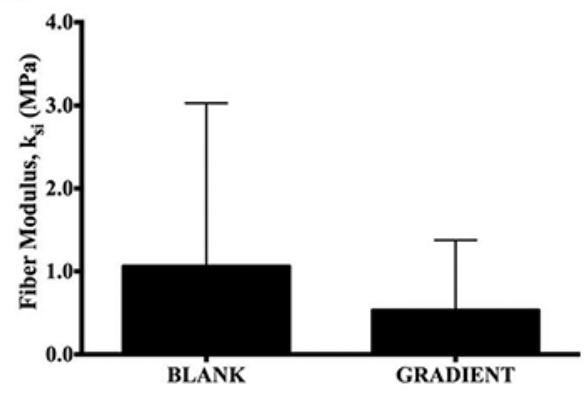

C

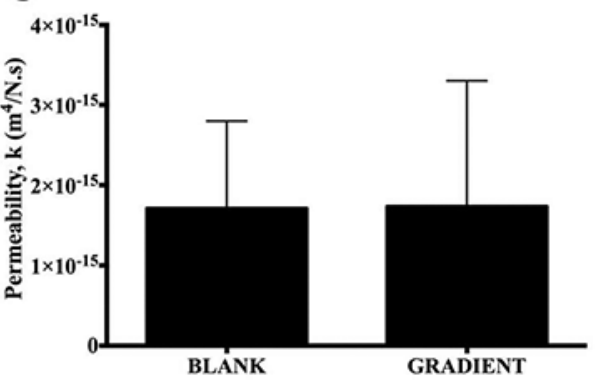

D

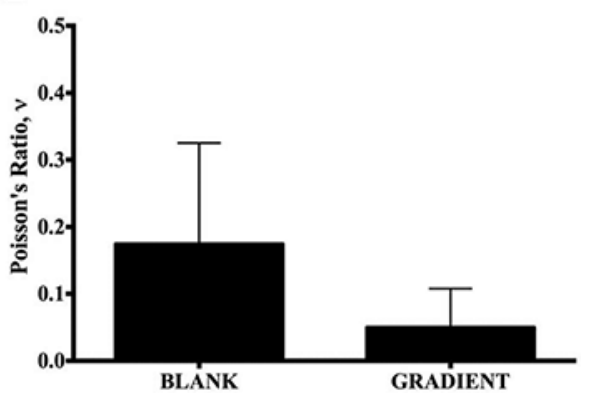

Figure 5.

Mechanical testing results of repair tissue in the retrieved joints from the BLANK and GRADIENT groups at 12 weeks postimplantation. (A) Elastic modulus, (B) fiber modulus, (C) permeability, and (D) Poisson's ratio. All values are expressed as the average + standard deviation $(n=4)$. No significant differences in any of the four model parameter values were observed between the two groups. The Poisson's ratio for the BLANK group was $0.17 \pm$ 0.15 and the GRADIENT group Poisson's ratio was $0.043 \pm 0.051$. 


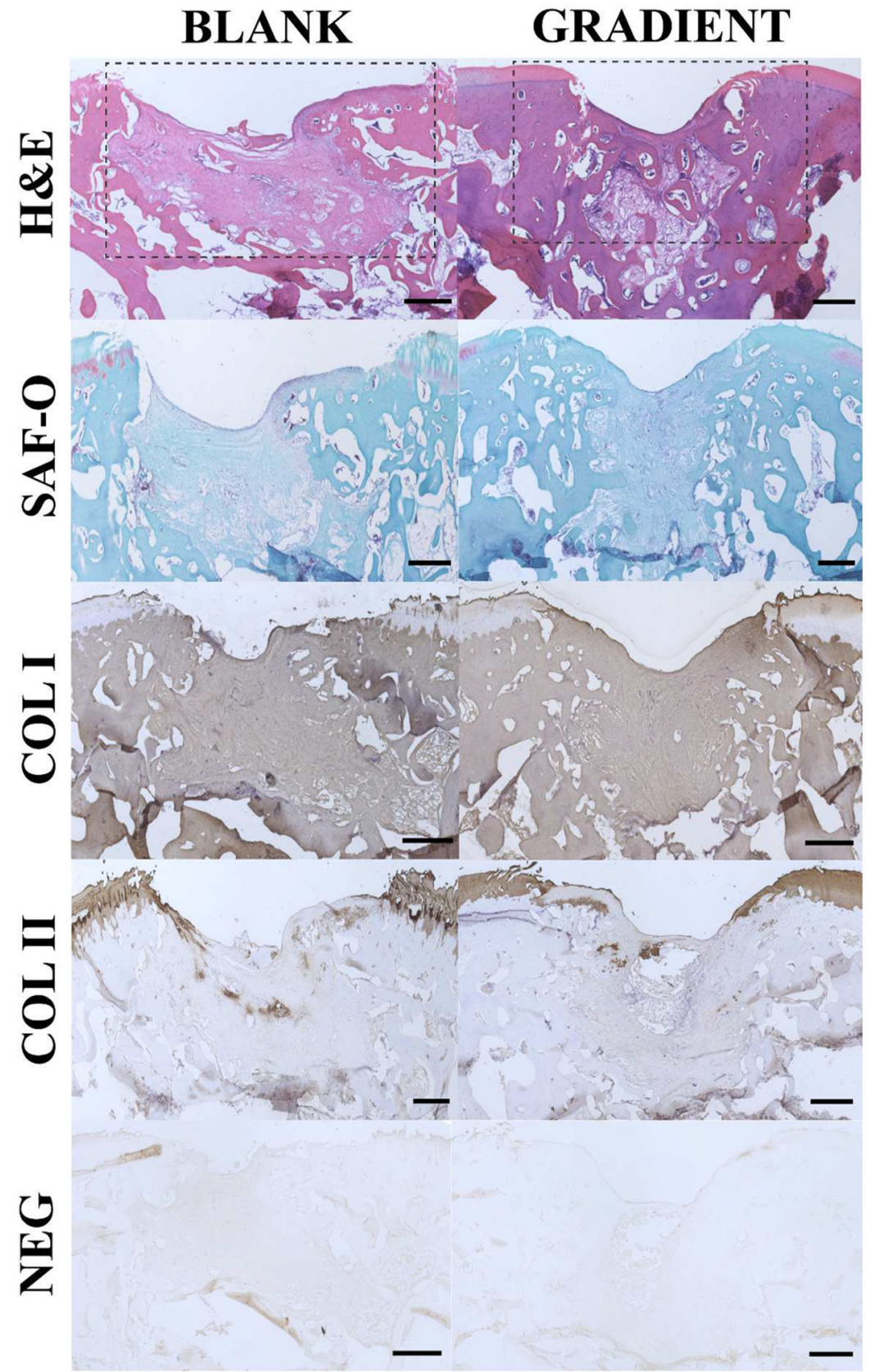

Figure 6.

Representative histological and immunohistochemical (IHC) staining images for the BLANK and GRADIENT groups. The sections were stained for hematoxylin and eosin, safranin-O, collagen I, and collagen II. Negative controls for IHC were also run with the primary antibody omitted. The boxes in the top row outline the defect area. The regenerated tissue in both of the groups was predominantly fibrous in nature with some evidence of cartilage repair and subchondral bone regeneration especially at the edges of the defect site. Scale bars: $500 \mu \mathrm{m}$. 


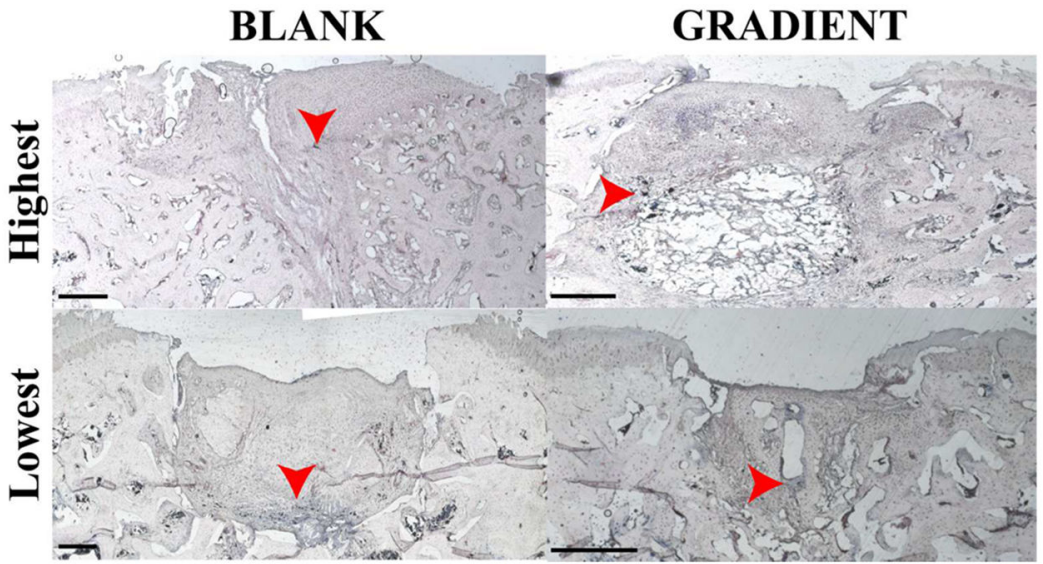

Figure 7.

Sudan black staining images for the implants that received the highest and lowest histological scores in both the BLANK and GRADIENT groups. The defect sites in both of the groups demonstrated mild staining overall with a few dark-staining spots (arrows). Scale bars: $500 \mu \mathrm{m}$. 
Table 1.

List of Implant Received by Each Animal and the Type of Analysis Performed for the Implants

\begin{tabular}{|c|c|c|c|c|}
\hline $\begin{array}{c}\text { animal } \\
\text { no. }\end{array}$ & left knee & right knee & $\underset{\text { (weeks) }}{\text { time }}$ & analysis \\
\hline rabbit 1 & BLANK & GRADIENT & 12 & morphology \& histology \\
\hline rabbit 2 & BLANK & GRADIENT & 12 & morphology \& histology \\
\hline rabbit 3 & BLANK & GRADIENT & 12 & morphology \& mechanical \\
\hline rabbit $4^{a}$ & BLANK & GRADIENT & 12 & euthanized prematurely \\
\hline rabbit 5 & BLANK & GRADIENT & 12 & morphology \& mechanical \\
\hline rabbit 6 & GRADIENT & BLANK & 12 & morphology \& histology \\
\hline rabbit 7 & GRADIENT & BLANK & 12 & morphology \& histology \\
\hline rabbit 8 & GRADIENT & BLANK & 12 & morphology \& mechanical \\
\hline rabbit 9 & GRADIENT & BLANK & 12 & morphology \& mechanical \\
\hline rabbit $10^{b}$ & GRADIENT & BLANK & 12 & euthanized prematurely \\
\hline
\end{tabular}

